\title{
Avaliação da Aprendizagem em Experimentos com Jogo Educativo de Engenharia de Requisitos
}

\author{
Rafael Q. Gonçalves, Marcello Thiry e Alessandra Zoucas \\ ${ }^{1}$ Universidade do Vale do Itajaí (UNIVALI) - Campus São José \\ Ciência da Computação \\ Rodovia SC 407, km 04, São José - SC - Brasil \\ rafael.q.g@hotmail.com \{thiry, azoucas\}@univali.br
}

\begin{abstract}
The Requirements Engineering process has been considered as the source of the main failures causes in software projects in enterprises. This situation occurs, among other reasons, because a great extent of the students who join the labor Market lack the necessary experience to perform the duties of a requirements analyst. As a consequence, the projects may not fulfill the clients' expectations, once the projects lack the application of good practices of requirements engineering. This study aims to evaluate empirically the learning of Requirements Engineering through an educational game. The game evaluation is presented from the execution of two experiments with undergraduate students, indicating that the use of educational games may contribute to the learning of Requirements Engineering.
\end{abstract}

Resumo. A Engenharia de Requisitos tem sido apontada como a origem das principais causas na falha de projetos de software em empresas. Isto vem ocorrendo, entre outros motivos, porque parte dos estudantes entra no mercado de trabalho sem a experiência necessária para exercer a função de analista de requisitos. Como consequência, projetos podem não atender as expectativas do cliente, uma vez que falta a aplicação de boas práticas da Engenharia de Requisitos. O objetivo deste trabalho é avaliar empiricamente a aprendizagem de Engenharia de Requisitos por meio de aplicações de um jogo educacional. A avaliação é apresentada a partir de dois experimentos com alunos de curso superior, indicando que o uso de jogos educativos pode contribuir para a aprendizagem de Engenharia de Requisitos.

\section{Introdução}

A Engenharia de Requisitos é um processo considerado por diversos autores como a parte mais crítica no desenvolvimento software, uma vez que a qualidade do produto final depende fortemente da qualidade dos requisitos [Ferguson and Lami 2006]. Existem trabalhos demonstrando que $85 \%$ dos problemas de software têm origem na atividade de elicitação de requisitos [Fernandes, Machado and Seidman 2009]. Tipicamente, após três a cinco anos de trabalho no desenvolvimento de software, os profissionais percebem que é necessário ter conhecimento aprofundado em tópicos da 
disciplina de Engenharia de Software [Fernandes, Machado and Seidman 2009]. Antes disso, tópicos como Engenharia de Requisitos, Gerenciamento de Projetos e Qualidade não são considerados relevantes para os profissionais, pois no início de suas carreiras, o foco maior está voltado para conhecimentos tecnológicos. Entretanto, para minimizar fracassos em projetos de desenvolvimento de software, é fundamental ter profissionais formados e treinados no processo de Engenharia de Requisitos, que sejam capazes de realizar a elicitação de requisitos com qualidade [Fernandes, Machado and Seidman 2009]. Deste modo, evidencia-se a necessidade de formar profissionais nas habilidades necessárias para execução deste processo [Romero, Vizcaíno and Piattini 2008].

O processo de Engenharia de Requisitos é dividido em quatro fases: i) identificação; ii) análise e negociação; iii) especificação e documentação; e iv) validação [Soares 2005], podendo ainda incluir a atividade de gerência de requisitos [Sommerville 2007]. Porém, o ensino nas universidades parece não ser suficiente para formar o aluno na execução desse processo. Um possível motivo está no modo de ensino que é, usualmente, voltado para teoria, raramente envolvendo os estudantes em projetos reais [Romero, Vizcaíno and Piattini 2008]. Outro motivo pode ser também a própria maturidade dos estudantes, que pelo momento no curso, podem ainda não estar trabalhando com projetos mais complexos que demonstrem as dificuldades da vida real. Nesta situação, o estudante não percebe a real importância de executar um processo de Engenharia de Requisitos adequado. Os dois motivos aqui apresentados sugerem que os desenvolvedores de software, depois que deixam a universidade, normalmente não estão preparados para executar as atividades referentes à Engenharia de Requisitos no contexto de empresas [Sommerville and Kotonya 1998].

Pesquisas na área de treinamento e educação sugerem o uso de jogos, pois podem engajar o estudante, reforçando conceitos através da prática, e aprofundando os conhecimentos [El-shamy 2001]. A utilidade desses jogos está altamente acoplada a sua habilidade de entreter os estudantes e refletir as atividades da Engenharia de Requisitos do mundo real, e de serem simples de entender e de jogar [Alexander and Beatty 2008]. $\mathrm{O}$ envolvimento emocional de um estudante aumenta conforme o entretenimento, e assim ocorre a variação de estímulos, o que ajuda o estudante a reter novos conhecimentos, e para isto as aulas palestradas não são suficientes [Alexander and Beatty 2008].

Este trabalho apresenta o desenvolvimento e o estudo empírico de aplicação deste jogo para apoiar o ensino de Engenharia de Requisitos em cursos de graduação em Ciência da Computação e de curso tecnólogo em Gestão de Tecnologia da Informação. O jogo foi utilizado para os estudantes reforçarem conceitos sobre Engenharia de Requisitos e aprofundarem seus conhecimentos, permitindo a prática do aprendizado através de um jogo lúdico, focando a facilidade de uso e de jogabilidade.

O jogo desenvolvido foi avaliado a partir de um estudo empírico constituído de um conjunto de dois experimentos. Em um primeiro momento foram definidas as hipóteses referentes ao aprendizado dos alunos com a utilização do jogo, a partir da taxonomia revisada de Bloom [Anderson 1999]. Cada estudo empírico foi composto de um pré-teste, aplicado antes dos alunos jogarem, e de um pós-teste realizado logo após o jogo. Ainda, a distribuição dos participantes nos dois grupos (grupo de controle e grupo experimental) foi feito de forma aleatória. Os alunos de ambos os grupos realizaram o 
pré e pós-teste, porém os alunos do grupo de controle não jogaram o jogo entre a realização de um teste e outro, com o objetivo de verificar a influência que jogo teve para o progresso no aprendizado dos alunos que o jogaram em relação aos outros alunos.

$\mathrm{Na}$ próxima seção, são apresentados dois jogos correlatos para ensino de Requisitos. A seção 3 apresenta o jogo desenvolvido "Ilha dos Requisitos". Os detalhes da avaliação da aprendizagem com o apoio do jogo são apresentados na seção 4. A seção 5 apresenta uma discussão dos resultados e as conclusões deste artigo.

\section{Trabalhos Correlatos}

Durante a execução deste trabalho, foi realizada uma pesquisa para encontrar jogos educativos para o ensino de Engenharia de Requisitos. A seleção dos jogos foi feita através da busca de artigos científicos que abordassem jogos educativos e preferencialmente focados no ensino de Engenharia de Requisitos.

Para encontrar os trabalhos correlatos foram utilizadas ferramentas de busca, tais como Google Scholar, CiteSeer e IEEExplorer em pesquisas utilizando combinações das seguintes sentenças: Requirements Engineering; Game; Educational game; Teaching e Game for teaching. Embora haja diversos jogos voltados para o ensino de Engenharia de Software e Gerência de Projetos, nesta pesquisa foram encontrados, publicados na literatura técnica, apenas dois jogos voltados especificamente para Engenharia de Requisitos: RE-O-Poly [Smith and Gotel 2008] e Software Quantum [Knauss, Schneider and Stapel 2008]. Assim, estes são dois trabalhos publicados na literatura técnica e que estão associados à mesma questão da pesquisa apresentada neste artigo.

\subsection{Re-O-Poly}

O jogo RE-O-Poly [Smith and Gotel 2008] é baseado no popular jogo de tabuleiro Banco Imobiliário. Seu objetivo é ensinar conhecimentos básicos sobre boas práticas de Engenharia de Requisitos para organizações que desenvolvem software. Durante uma partida, os jogadores precisam responder perguntas sobre Engenharia de Requisitos, sendo que algumas são sobre conhecimentos gerais e outras sobre a aplicação dos conhecimentos de Engenharia de Requisitos em situações específicas de um determinado projeto.

O tabuleiro do jogo é dividido em quatro partes, que simbolizam os quatros estágios básicos do processo de Engenharia de Requisitos, que são: elicitação, análise e validação, documentação, e gerenciamento de mudanças. E devido a esta divisão, uma rodada através do tabuleiro representa uma passagem pelo processo de Engenharia de Requisitos, e o avanço do jogador no tabuleiro ocorre através de jogada de um dado, onde o resultado do dado indica a quantidade de casas que o jogador deve avançar no tabuleiro.

\subsection{Software Quantum}

O Software Quantum [Knauss, Schneider and Stapel 2008] é um jogo de simulação disponível na Internet (se.uni-hannover.de/en/qgame), apresentado em uma applet Java. O jogo foi desenvolvido para conscientizar os estudantes da importância da Engenharia de Requisitos, mostrando que quando as etapas do processo de Engenharia de 
Requisitos não são realizadas corretamente, dificilmente o resultado final do projeto irá atender as expectativas do cliente. Em relação à aprendizagem, o jogo foi projetado para ensinar os seguintes conteúdos de Engenharia de Requisitos:

- Lembrar que os Requisitos necessitam passar por um fluxo entre o cliente e o código fonte, envolvendo todos os stakeholders do projeto.

- Lembrar que requisitos devem ser documentados, pois assim eles têm maior durabilidade, pois conhecimento tácito tende a ser perdido.

- Lembrar que existe mais de uma possibilidade para trilhar o caminho entre a solicitação do cliente e a geração código fonte, sendo que alguns caminhos podem optar por realizar uma melhor documentação, e outros uma documentação menos completa, e que também pode haver uma combinação de técnicas, com documentação em alguns momentos e comunicação informal em outros.

- Lembrar que é importante validar a informação recebida, pois quando a informação não é validada, os requisitos podem ser interpretados de maneira incorreta, ou pode haver perda dos requisitos originais, e até mesmo a implementação de requisitos não solicitados.

\subsection{Análise dos Trabalhos Correlatos}

Ambos os jogos avaliados possuem os objetivos de aprendizagem definidos. Foi possível observar indícios de que jogos não digitais propõem atividades cognitivas para o aprendizado dos jogadores, já jogos digitais utilizam o comportamentalismo para ensinar aos jogadores. Com relação à taxonomia de Bloom, uma vez que o nível da taxonomia pode variar para cada objetivo de aprendizagem, os jogos foram classificados em relação ao nível com maior ocorrência. Desta forma, o jogo RE-O-Poly foi classificado com o nível "Aplicar", já o jogo Software Quantum classificado com o nível "Lembrar". Quanto ao gênero ambos os jogos foram classificados como jogo de simulação. Os dois jogos avaliados ensinam processos ou teorias, o que parece demonstrar que grande parte da literatura sobre ensino de Engenharia de Requisitos está focada no ensino de processos e conceitos.

Nenhum dos trabalhos estudados apresenta um estudo empírico sobre sua efetividade no processo de aprendizagem. Neste sentido, este trabalho procura contribuir com uma avaliação empírica da aprendizagem, comparando o jogo educativo "A Ilha dos Requistos" a diferentes métodos de ensino nos moldes de outros trabalhos publicados deste mesmo grupo de pesquisa [Kochanski, Wangenheim and Thiry 2009].

\section{O Jogo Educativo "A Ilha dos Requisitos"}

Este trabalho envolveu a construção do jogo educativo "A Ilha dos Requistos", cujo objetivo é auxiliar no ensino dos principais conceitos envolvidos na área de conhecimento Engenharia de Requisitos, tais como:

- o processo de Engenharia de Requisitos;

- o papel e as responsabilidades do analista de requisitos;

- os tipos de requisitos. 
O jogo também tem como finalidade transmitir ao jogador a importância de aplicar adequadamente a Engenharia de Requisitos em projetos de software. O jogo é contextualizado em um ambiente lúdico, com o objetivo de entreter os jogadores, através de seu enredo e dos personagens. Durante uma partida, o jogador deverá realizar desafios, onde são avaliados seus conhecimentos em Engenharia de Requisitos. Porém, os conteúdos abordados na Engenharia de Requisitos estão imersos no contexto lúdico do jogo, com a finalidade de minimizar o impacto da interação do jogador com o conteúdo formal desta área de conhecimento.

O objetivo do jogo é ajudar o personagem Jack Reqs, a fugir da "A Ilha dos Requistos". A ilha, habitada por tribos canibais, tem um vulcão que esta prestes a entrar em erupção. O objetivo é ajudar Jack a escapar da ilha antes que o vulcão exploda a ilha. Para alcançar este objetivo, o jogo é baseado num conjunto de regras:

- O jogo é em $3^{a}$ pessoa, onde o jogador ajudará o personagem Jack Reqs a enfrentar os desafios para escapar da Ilha dos Requisitos antes que o vulcão BigBug entre em erupção.

- O jogo deve ser terminado em no máximo 45 minutos. Esta duração representa 22 dias na vida real ou as 30 luas da profecia Nerd. Após este período de tempo, o vulcão entrará em erupção.

- O mapa do jogo é um mapa estilizado da ilha que irá apontar diferentes locais. Cada local representa um desafio para o jogador.

- Eventualmente, alguns locais estão desabilitados até que o jogador atinja certo ponto do jogo.

- Sempre que o jogador não conseguir ajudar Jack a resolver um desafio, a penalidade é no tempo do jogo. Jack perderá dias valiosos (na verdade, minutos no tempo real do jogo) para completar o objetivo.

- O jogador coleta pontos durante os desafios, e estes pontos são contabilizados de acordo com o tempo gasto para execução de cada desafio e pela quantidade de erros cometidos.

- O jogador pode consultar o notebook de Jack durante o jogo para pegar dicas sobre um desafio. Entretanto, a bateria do notebook não está totalmente carregada. Assim, o jogador pode contar com uma quantidade limitada de acessos durante o jogo. Desta forma, ele deverá utilizá-lo com sabedoria.

A Figura 1 apresenta uma tela do jogo "A Ilha dos Requisitos", que mostra o mapa do jogo, onde o jogador navega entre os desafios. Esta tela apresenta o tempo decorrido desde o início da partida e a área do mapa onde o jogador se encontra. 


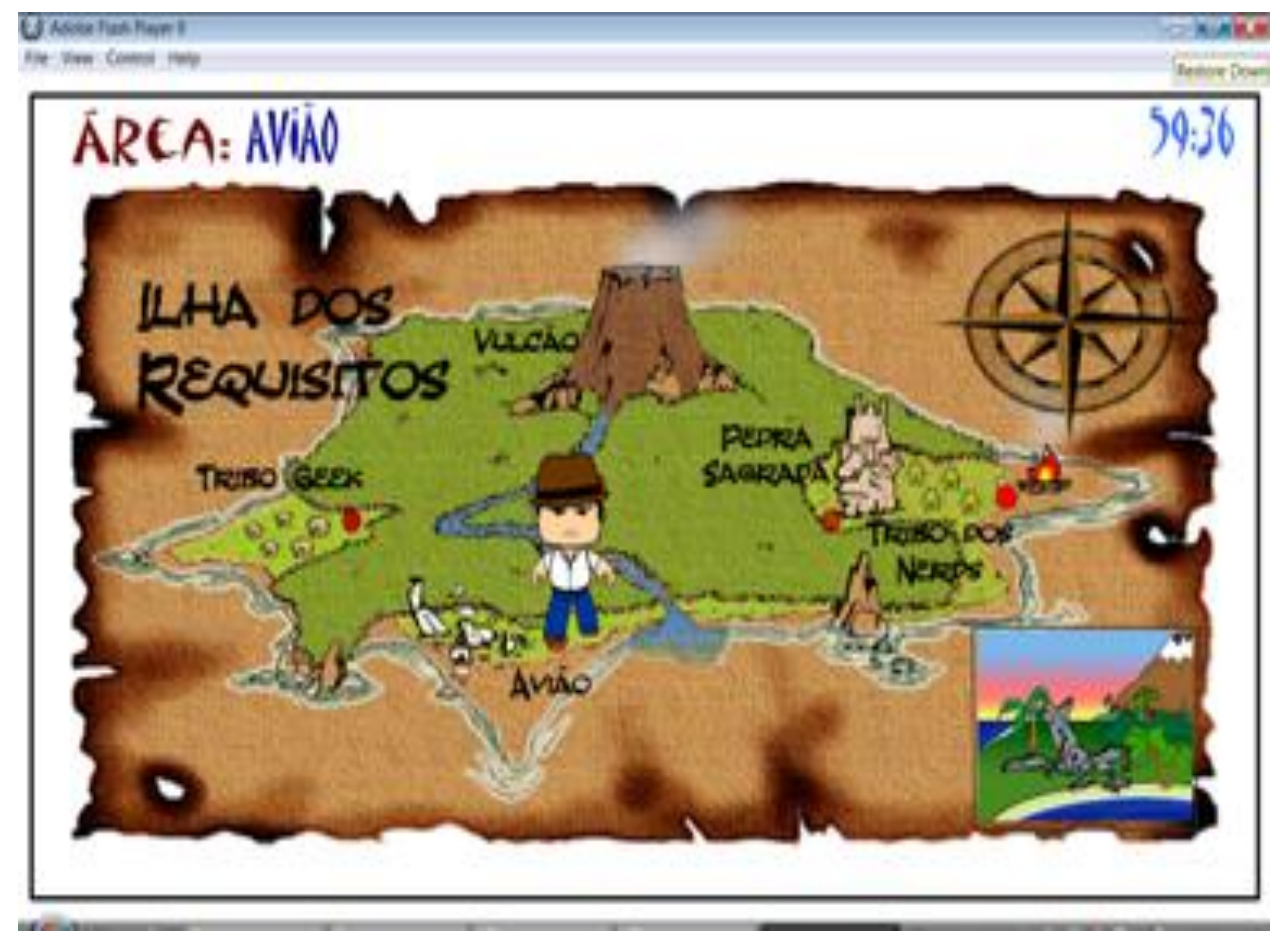

Figura 1- Tela do jogo "A llha dos Requisitos".

\subsection{Classificação do jogo}

O jogo é classificado com relação aos seguintes aspectos:

- Gênero: "Aventura" e "Estratégia", pois apresenta ao jogador, como nos jogos de aventura, um mundo novo e inexplorado onde são apresentados desafios que devem ser resolvidos com objetos coletados durante o jogo. Ainda permite que o jogador, como nos jogos de estratégia, solicite algo que será construído, se abstraindo dos detalhes.

- Interação: por meio de um navegador web o jogo reage aos comandos executados pelo jogador através de mudança do comportamento da tela ou através dos feedbacks. O jogador tem também uma interação indireta com outros jogadores, através de um ranking de pontuações que exibe o resultado de todos os jogadores que já jogaram "A Ilha dos Requisitos".

- Plataforma: flash player, que além de ser portável em diversos navegadores web proporciona recursos para desenvolver os mecanismos de interação entre o jogador e o jogo.

\subsection{Desafios}

Durante o jogo "A Ilha dos Requisitos" o jogador enfrenta uma série de desafios, onde cada desafio está relacionado a um conteúdo da Engenharia de Requisitos. Foram desenvolvidos sete desafios, organizados de modo que o jogador faça uma passagem completa por todas as fases do processo de Engenharia de Requisitos [Thiry, Zoucas and Goncalves 2010] [Goncalves and Thiry 2010]. 
A Tabela 1 apresenta os conteúdos da Engenharia de Requisitos que são abordados em cada desafio do jogo, e uma breve descrição da atividade que o jogador deverá realizar durante desafio.

Tabela 1 - Desafios do jogo

\begin{tabular}{|l|l|l|}
\hline $\mathbf{N}^{\mathbf{0}}$ & Conteúdo abordado & Descrição \\
\hline 1 & $\begin{array}{l}\text { Processo de Engenharia de } \\
\text { Requisitos }\end{array}$ & $\begin{array}{l}\text { O jogador deve ordenar as fases do processo de Engenharia } \\
\text { de Requisitos. }\end{array}$ \\
\hline 2 & $\begin{array}{l}\text { Os papeis } \\
\text { responsabilidades do analista } \\
\text { de requisitos. }\end{array}$ & $\begin{array}{l}\text { O jogador deve marcar quais as habilidades são do analista } \\
\text { de requisitos. }\end{array}$ \\
\hline 3 & Análise do problema & O jogador deve distinguir o problema de possíveis soluções. \\
\hline 5 & Classificação dos requisitos & $\begin{array}{l}\text { O jogador deve classificar um conjunto de requisitos, cada } \\
\text { um em uma das duas possíveis categorias: funcionais e não } \\
\text { funcionais. }\end{array}$ \\
\hline 6 & $\begin{array}{l}\text { O processo de mudança dos } \\
\text { requisitos }\end{array}$ & $\begin{array}{l}\text { O jogador deve levar os requisitos ao "cliente" para que } \\
\text { estes sejam validados antes do início da execução. }\end{array}$ \\
\hline 7 & Gerência dos requisitos & $\begin{array}{l}\text { O jogador deve ordenar o processo de mudança dos } \\
\text { requisitos. }\end{array}$ \\
\hline
\end{tabular}

Os desafios estão ligados ao contexto da estória do jogo. Portanto, os conteúdos abordados em cada desafio estão envolvidos por um conjunto de elementos lúdicos, com o objetivo de diminuir o impacto da apresentação do conteúdo formal da Engenharia de Requisitos para o aluno, e de tornar este contato mais sutil e prazeroso.

Um exemplo de desafio é apresentado na Figura 2 que ilustra o primeiro desafio do jogo, onde o jogador deve colocar as fases do processo de Engenharia de Requisitos na ordem correta. As fases são representadas por runas (pedras usadas para ler a sorte na cultura nórdica), inicialmente, dispostas em ordem aleatória. Com relação aos aspectos lúdicos, neste desafio o jogador está sujeito a ser devorado pela tribo de canibais, denominada tribo dos NERDS, caso não consiga convencê-los de que conhece o processo de Engenharia de Requisitos.

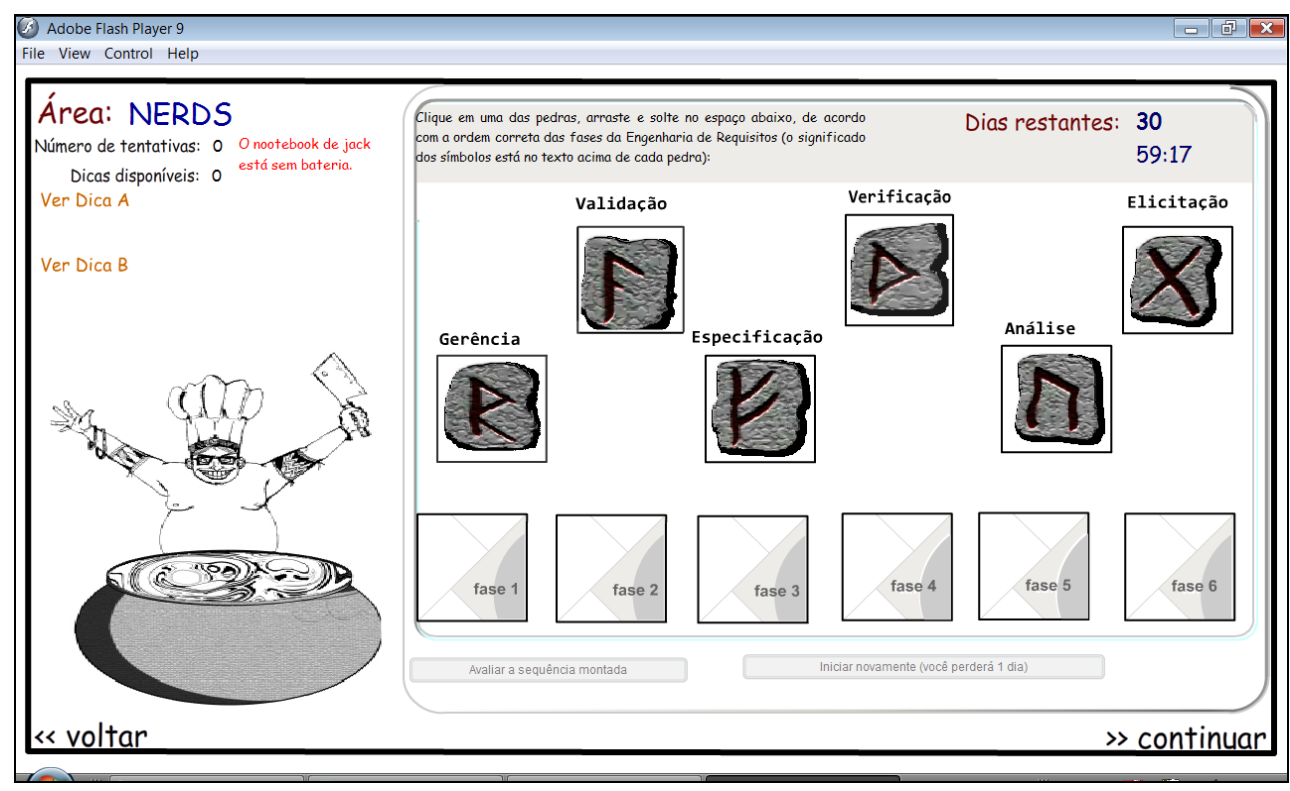

Figura 2 - Desafio 1 


\subsection{Design Instrucional}

A Tabela 2 apresenta os tópicos que o jogo visa reforçar o aprendizado, e a qual nível da taxonomia revisada de Bloom cada tópico está associado [Thiry, Zoucas and Goncalves 2010] [Goncalves and Thiry 2010].

Tabela 2 - Design Instrucional

\begin{tabular}{|l|l|l|}
\hline $\mathbf{N}^{\mathbf{0}}$ & Tópico sobre requisitos & Nível na Taxonomia de Bloom \\
\hline 1 & Processo de Engenharia de Requisitos & Lembrar \\
\hline 2 & Validação dos requisitos & Lembrar \\
\hline 3 & Papel do analista de requisitos & Lembrar \\
\hline 4 & Estudo de viabilidade & Lembrar \\
\hline 5 & Especificação dos requisitos & Entender \\
\hline 6 & Classificação dos requisitos & Entender \\
\hline 7 & Verificação & Lembrar \\
\hline 8 & Documentação dos requisitos & Entender \\
\hline 9 & Gerência dos requisitos & Lembrar \\
\hline
\end{tabular}

Para promover o aprendizado, os tópicos apresentados no design instrucional, foram utilizadas técnicas comportamentalistas [Greeno, Collins and Resnick 1996], pois através da avaliação dos jogos educativos, foi concluído que para jogos digitais, as técnicas comportamentalistas são mais fáceis de serem aplicadas, devido à limitação de interatividade entre o jogador e a máquina.

\section{Avaliação da Aprendizagem}

\subsection{O Estudo Empírico}

O objetivo do estudo empírico foi de responder se "O jogo 'A Ilha dos Requisitos' contribui para o aprendizado dos alunos?". Para tal, foram consideradas as seguintes hipóteses:

- H0: Não há diferença com relação ao nível de lembrança, conhecimento, compreensão e aplicação entre uma abordagem de ensino apoiada pelo jogo "A Ilha dos Requisito" e uma abordagem de ensino sem este apoio.

- H1: a efetividade de aprendizagem nos níveis de conhecimento, compreensão e aplicação do grupo experimental são superiores aos do grupo de controle.

- H2: a aplicação do jogo "A Ilha dos Requisitos" torna o processo de ensino aprendizagem mais atrativo.

Para a realização do estudo empírico foi realizado um conjunto de dois experimentos com alunos de curso superior em Ciência da Computação e de curso tecnólogo em Gestão de Tecnologia da Informação. Os experimentos produziram avaliações quantitativas e também qualitativas com o objetivo de medir o conhecimento adquirido após sua aplicação. A estrutura do experimento seguiu a proposta definida por Kochanski [Kochanski, Wangenheim and Thiry 2009]. O estudo empírico consistiu nos seguintes passos: 
1. Preparação das avaliações: a avaliação foi preparada, visando abranger todos os conteúdos que são contemplados no jogo.

2. Assinatura do acordo de participação: os alunos assinaram um acordo demonstrando interesse em participar do experimento.

3. Preenchimento do questionário de perfil: os alunos preencheram um questionário com perguntas sobre suas experiências e seu conhecimento prévio no assunto para que fosse possível estabelecer um background de cada aluno e dos grupos.

4. Preparação dos alunos: Os alunos envolvidos no experimento receberam aulas teóricas sobre Engenharia de Requisitos antes de jogar o jogo.

5. Aplicação da primeira avaliação: todos os alunos realizaram a primeira avaliação para medir seus conhecimentos após a aula teórica.

6. Formação dos grupos: os alunos foram divididos em dois grupos, um grupo de controle e um grupo experimental.

7. Aplicação do jogo: nos dois experimentos os alunos do grupo experimental jogaram o jogo, os alunos do grupo de controle jogaram outro jogo sem nenhuma relação com Engenharia de Requisitos (este jogo foi considerado como um placebo).

8. Aplicação da segunda avaliação: os alunos de ambos os grupos realizaram a segunda avaliação.

9. Análise dos resultados: utilizando os dados coletados na primeira e na segunda avaliação foram aplicados testes de hipótese para verificar se houve melhora no desempenho dos alunos após a prática do jogo.

10. Aplicação de um questionário: os alunos responderam perguntas relacionadas com sua percepção do jogo. Este questionário serviu como base para uma avaliação qualitativa do jogo e da efetividade de aprendizagem.

As hipóteses $\mathbf{H 0}$ e $\mathbf{H 1}$ foram avaliadas a partir do teste estatístico comentado no passo 8. A hipótese $\mathbf{H 2}$ foi estabelecida a partir da avaliação qualitativa baseada no questionário respondido pelos alunos no passo 10 .

\subsection{Dados Coletados}

Para os dois experimentos, houve a preocupação com a precisão e com o problema da interpretação estatística sobre amostras pequenas. Deste modo, foi utilizado um teste não paramétrico (Mann-Whitney unilateral U) para avaliar as hipóteses sobre a efetividade de aprendizagem do jogo. Este teste é considerado a alternativa mais poderosa para o teste paramétrico t para amostras independentes [Levin and Fox 2006]. Portanto, foram construídas as Tabelas 3 e 4 que apresentam a classificação dos participantes de experimento pela diferença das pontuações realizadas entre pré e pósteste. Para chegar à classificação apresentada, as diferenças (nota no pós-teste menos a nota no pré-teste) foram colocadas em ordem ascendente e a classificação iniciada em 1 foi atribuída a partir do menor resultado. No caso de haverem duas ou mais diferenças iguais, a classificação foi obtida através do cálculo da média das sequências das mesmas. $\mathrm{O}$ primeiro experimento contou com a participação de 31 alunos. 
Tabela 3 - Design Instrucional (dados coletados no primeiro experimento)

\begin{tabular}{|c|c|c|c|}
\hline Sequência & Diferença & Classificação & Grupo \\
\hline 1 & -3 & 1 & Controle \\
\hline 2 & -2 & 4,5 & Controle \\
\hline 3 & -2 & 4,5 & Controle \\
\hline 4 & -2 & 4,5 & Controle \\
\hline 5 & -2 & 4,5 & Controle \\
\hline 6 & -2 & 4,5 & Controle \\
\hline 7 & -2 & 4,5 & Experimental \\
\hline 8 & -1 & 7,5 & Controle \\
\hline 9 & -1 & 7,5 & Controle \\
\hline 10 & -1 & 7,5 & Controle \\
\hline 11 & -1 & 7,5 & Experimental \\
\hline 12 & -1 & 7,5 & Experimental \\
\hline 13 & 0 & 14,5 & Controle \\
\hline 14 & 0 & 14,5 & Controle \\
\hline 15 & 0 & 14,5 & Experimental \\
\hline 16 & 0 & 14,5 & Experimental \\
\hline 17 & 1 & 19,5 & Controle \\
\hline 18 & 1 & 19,5 & Controle \\
\hline 19 & 1 & 19,5 & Controle \\
\hline 20 & 1 & 19,5 & Experimental \\
\hline 21 & 1 & 19,5 & Experimental \\
\hline 22 & 1 & 19,5 & Experimental \\
\hline 23 & 2 & 25 & Experimental \\
\hline 24 & 2 & 25 & Experimental \\
\hline 25 & 2 & 25 & Experimental \\
\hline 26 & 2 & 25 & Experimental \\
\hline 27 & 2 & 25 & Experimental \\
\hline 28 & 3 & 28,5 & Controle \\
\hline 29 & 3 & 28,5 & Experimental \\
\hline 30 & 4 & 30,5 & Experimental \\
\hline 31 & 4 & 30,5 & Experimental \\
\hline
\end{tabular}

O segundo experimento contou com a participação de 13 alunos. Os dados coletados neste experimento estão apresentados na Tabela 4.

Tabela 4 - Design Instrucional (dados coletados no segundo experimento)

\begin{tabular}{|l|l|l|l|}
\hline Sequência & Diferença & Classificação & Grupo \\
\hline 1 & -1 & 1 & Controle \\
\hline 2 & 0 & 3,5 & Controle \\
\hline 3 & 0 & 3,5 & Controle \\
\hline 4 & 0 & 3,5 & Controle \\
\hline 5 & 1 & 5,5 & Experimental \\
\hline 6 & 1 & 5,5 & Controle \\
\hline 7 & 2 & 7,5 & Controle \\
\hline 8 & 2 & 7,5 & Experimental \\
\hline 9 & 3 & 10,5 & Experimental \\
\hline 10 & 3 & 10,5 & Experimental \\
\hline 11 & 3 & 10,5 & Experimental \\
\hline 12 & 3 & 10,5 & Experimental \\
\hline 13 & 5 & 13 & Experimental \\
\hline
\end{tabular}




\subsection{Avaliação da Aprendizagem com aplicação do Jogo A Ilha dos Requisitos}

Para aplicar o teste estatístico Mann-Whitney, foi calculado o valor das variáveis T1 e $\mathrm{T} 2$, que correspondem respectivamente ao somatório das classificações referentes aos elementos do grupo experimental e de controle. Desta forma, tem-se para o primeiro experimento $\mathrm{T} 1=321,5$ e $\mathrm{T} 2=162$.

Depois de calculados os valores de T1 e T2, foi selecionada a variável de maior resultado. Neste caso, o resultado da maior classificação é T1 com valor 321,5. T1 foi então utilizada na variável Tx da fórmula do cálculo do valor de U. Os valores de n1, n2 e nx foram obtidos da seguinte forma:

- $\mathrm{n} 1$ : número de participantes do grupo experimental;

- n2: número de participantes do grupo de controle; e

- nx: número de participantes do grupo com maior somatório de classificação.

No caso do primeiro experimento realizado o valor de $\mathrm{n} 1=16, \mathrm{n} 2=15$ e $\mathrm{nx}=$ 16, pois havia 16 participantes no grupo experimental (n1), 15 participantes do grupo de controle (n2) e o grupo com maior somatório de classificação (nx) foi o grupo experimental com 16 participantes. De posse dos valores destas variáveis, foi realizado o cálculo de U pela equação:

$$
\mathrm{U}=\mathrm{n} 1 * \mathrm{n} 2+\mathrm{nx} *(\mathrm{nx}+1) / 2-\mathrm{Tx}
$$

Atribuindo os valores às respectivas variáveis em (1) tem-se:

$$
\mathrm{U}=15 * 16+16 *(16+1) / 2-321,5
$$

$\mathrm{O}$ valor calculado para $U$ foi $\mathbf{5 4 , 5}$. O passo seguinte foi utilizar a tabela de valores críticos de U para o teste de Mann-Whitney. O valor da interseção entre n1=15 e $\mathrm{n} 2=16$ é 70 [Levin and Fox 2006].

O mesmo cálculo é realizado com os dados coletados durante o segundo experimento. Desta forma, tem-se para o segundo experimento $\mathrm{T} 1=68$ e $\mathrm{T} 2=24,5$. Neste caso, o resultado da maior classificação é T2 com valor 68 . Os valores de n1, n2 e nx obtidos foram respectivamente 7,6,7. Atribuindo os valores às respectivas variáveis em (1) tem-se:

$$
\mathrm{U}=7 * 6+7 *(7+1) / 2-68
$$

$\mathrm{O}$ valor calculado para $\mathrm{U}$ foi 2. O passo seguinte foi utilizar a tabela de valores críticos de U para o teste de Mann-Whitney. O valor crítico obtido na interseção entre n1=7 e n2=6 é 6 [Levin and Fox 2006]. Para que o resultado do teste seja significativo, o valor de $U$ obtido deve ser igual ou menor que o valor crítico da tabela [Levin and Fox 2006]. Analisando o resultado obtido por meio da aplicação dos dois experimentos, pode-se concluir que a hipótese $\mathbf{H 0}$ foi rejeitada com $95 \%$ de confiança em ambos os casos. Assim, pode-se inferir que a efetividade de aprendizagem relativa entre os grupos experimental e de controle são significativamente diferentes [Thiry et al 2010]. Deste modo, é possível afirmar com $95 \%$ de confiança que o jogo "A Ilha dos Requisitos" contribuiu para o ensino de Engenharia de Requisitos nos níveis de lembrança e entendimento. 
Para estabelecer a hipótese $\mathbf{H 2}$ (o jogo torna o processo de ensino-aprendizagem mais atrativo) foi formulado um questionário de percepção do jogo, onde foram apresentadas dez questões para verificar outros aspectos que não apenas a melhora do aprendizado dos alunos nos experimentos realizados. O objetivo foi identificar, de acordo com a percepção dos alunos, quanto o jogo pode apoiar o ensino como uma atividade motivante. Os 21 alunos que responderam este questionário informaram para cada pergunta um valor de 1 a 4 correspondendo respectivamente a (1) "Não", (2) "Mais para não", (3) "Mais para sim" e (4) "Sim".

A primeira coluna da Tabela 5 apresenta as dez perguntas deste questionário. As demais colunas mostram os resultados obtidos com o preenchimento do questionário pelos participantes tanto do primeiro quanto do segundo experimento. A última linha da Tabela 5 apresenta o total de cada resposta dada pelos alunos que responderam $o$ questionário.

Tabela 5 - Dados coletados na aplicação do questionário de percepção do jogo

\begin{tabular}{|c|c|c|c|c|}
\hline Perguntas do Qauestionário de Percepção do Jogo. & $\begin{array}{l}\text { Não } \\
(1)\end{array}$ & $\begin{array}{c}\text { Mais } \\
\text { para } \\
\text { Não } \\
\text { (2) }\end{array}$ & $\begin{array}{c}\text { Mais } \\
\text { para } \\
\text { sim } \\
(3) \\
\end{array}$ & $\begin{array}{l}\text { Sim } \\
(4)\end{array}$ \\
\hline 1. Você gostou do jogo? & $4,8 \%$ & $9,5 \%$ & $19,0 \%$ & $66,7 \%$ \\
\hline $\begin{array}{l}\text { 2. Os objetivos de aprendizagem foram atingidos com } \\
\text { o jogo? }\end{array}$ & $0,0 \%$ & $14,3 \%$ & $52,4 \%$ & $33,3 \%$ \\
\hline $\begin{array}{l}\text { 3. O conteúdo do jogo foi relevante para o } \\
\text { aprendizado? }\end{array}$ & $4,8 \%$ & $4,8 \%$ & $33,3 \%$ & $57,1 \%$ \\
\hline 4. A sequência de tópicos do jogo foi adequada? & $0,0 \%$ & $19,0 \%$ & $19,0 \%$ & $61,9 \%$ \\
\hline $\begin{array}{l}\text { 5. O jogo forneceu informação suficiente sobre o } \\
\text { assunto? }\end{array}$ & $4,8 \%$ & $38,1 \%$ & $28,6 \%$ & $28,6 \%$ \\
\hline $\begin{array}{l}\text { 6. O grau de dificuldade do jogo foi adequado para o } \\
\text { aprendizado? }\end{array}$ & $9,5 \%$ & $19,0 \%$ & $23,8 \%$ & $47,6 \%$ \\
\hline 7. A duração do jogo foi adequada? & $14,3 \%$ & $9,5 \%$ & $33,3 \%$ & $42,9 \%$ \\
\hline 8. O método de ensino do jogo foi adequado? & $0,0 \%$ & $14,3 \%$ & $23,8 \%$ & $61,9 \%$ \\
\hline $\begin{array}{l}\text { 9. A contextualização apresentada pelo jogo foi } \\
\text { adequada? }\end{array}$ & $0,0 \%$ & $14,3 \%$ & $23,8 \%$ & $61,9 \%$ \\
\hline Total: & $4,2 \%$ & $15,9 \%$ & $28,6 \%$ & $51,3 \%$ \\
\hline
\end{tabular}

Como pode ser observado na Tabela 5, mais de 90\% dos alunos responderam positivamente ("Sim" ou "Mais para sim") quando questionados sobre o conteúdo do jogo ser relevante para o aprendizado. Além disso, mais de $80 \%$ por cento dos alunos responderam de forma positiva quando questionados sobre terem gostado do jogo e sobre os objetivos de aprendizagem terem sido alcançados. Também, mais de $80 \%$ dos alunos afirmam positivamente que o jogo apresenta de forma adequada a sequência de tópicos, seu método de ensino e a contextualização apresentada. Quando questionados sobre o grau de dificuldade e duração do jogo, mais de $70 \%$ dos alunos se posicionou 
positivamente selecionando o valor 3 ("Mais para sim") ou 4 ("Sim") para a responder a pergunta. A questão sobre o jogo ter fornecido informação suficiente sobre o assunto foi a que obteve a menor freqüência de respostas positivas e ainda assim apresentou um número considerável de respostas positivas (57\%). Na última linha da Tabela 5 é possível observar, entre todas as perguntas do questionário, qual a resposta que foi dada com maior frequência pelos alunos que participaram do experimento. Assim, entre 189 respostas coletadas, 4,2\% alunos selecionaram o valor 1 ("Não"), 15,9\% dos alunos selecionaram o valor 2 ("Mais para não"), 28,6\% dos alunos selecionaram o valor 3 ("Mais para sim") e 51,3\% dos alunos selecionaram o valor 4 ("Sim").

Analisando os dados coletados, como resultado dos dois experimentos, foi possível verificar indícios de que o jogo, além de promover uma melhoria significativa na aprendizagem dos alunos que participaram da pesquisa, este foi avaliado pelos alunos de modo positivo em ambos experimentos. Assim, as respostas dos alunos indicam a possibilidade do jogo tornar o processo de aprendizado mais atrativo.

\section{CONCLUSÃO}

A aplicação dos experimentos para avaliar o jogo "A Ilha dos Requisitos" permitiu avaliações tanto quantitativas quanto qualitativas sobre o jogo. Durante a análise quantitativa dos dois experimentos, foi possível obter indícios de que o jogo contribui para a efetividade de aprendizagem de Engenharia de Requisitos nos níveis de lembrança e entendimento. Vale ressaltar que os resultados obtidos até o momento, mesmo que dentro do rigor estatístico, não podem ser generalizados.

Entre as principais ameaças da avaliação, deve-se considerar que os experimentos foram aplicados com a participação de alunos de curso superior em Ciência da Computação e de curso tecnólogo em Gestão de Tecnologia da Informação dentro de apenas duas instituições de ensino, onde os professores eram do mesmo grupo de pesquisa. Desta forma, é necessário ampliar o conjunto de experimentos para que seja possível avaliar as tendências de aprendizagem.

Como ameaça a construção do experimento está o fato de que os alunos previamente selecionados para participação do experimento não se façam presentes no momento de aplicação ou mesmo não completem todo o ciclo do experimento, participando apenas, por exemplo, a aplicação do pré-teste. Esta ameaça foi minimizada pelo fato de que os alunos foram previamente avisados que na data planejada para o experimento uma atividade seria realizada e a participação dos alunos nesta atividade seria utilizada pelo professor na pontuação da participação do aluno em aula.

Além disso, outras ameaças foram tratadas nos experimentos, de modo que elas fossem minimizadas. Por exemplo, a preocupação com a desigualdade no nível de conhecimento e experiência dos alunos em Engenharia de Requisitos foi tratada com a avaliação prévia do questionário de perfil dos alunos, respondido antes dos experimentos. Neste caso, não foram encontrados alunos que poderiam representar uma ameaça aos resultados obtidos.

Como ameaça interna à validade dos resultados foi levantada a hipótese dos alunos estudarem entre o intervalo do pré-teste e pós-teste. Esta ameaça foi eliminada, já que as atividades foram realizadas em sequência. 
A proximidade do pré-teste e pós-teste, estratégia utilizada para evitar que os alunos estudassem além do controle do experimento, apresentou outra ameaça referente ao fato de que o aluno pode ter respondido ao pós-teste apenas com base em sua memória de curto prazo. Entretanto, respostas com base na memória de curto prazo, não necessariamente representam o conhecimento que será efetivamente absorvido, uma vez que este pode simplesmente ser descartado. Como esta ameaça não havia sido inicialmente identificada, seu tratamento não foi realizado, ou seja, com base nos resultados atuais desta pesquisa não é possível afirmar quanto do novo conhecimento adquirido foi efetivamente absorvido pelos alunos.

Para a ameaça interna de incompatibilidade entre o pré-teste e pós-teste foi adotado o procedimento em que os dois instrumentos teriam as mesmas questões com ordenação diferente e algumas destas ainda com alterações nos dados das questões, sugerindo ao aluno verificar novamente a questão antes de assinalar a resposta correta. Para evitar que os alunos pudessem confundir ou mesmo não identificar, por exemplo, que as alternativas de uma dada pergunta tivessem sido reordenadas, na entrega do pósteste a turma foi informada que o instrumento era diferente do anterior e exigiria atenção para evitar confusão ao assinalar uma alternativa. Este procedimento claramente poderia ser evitado com dois testes completamente diferentes, o que poderia acarretar em uma ameaça maior que é a de incompatibilidade entre os instrumentos, ou seja, um destes ser mais simples ou mais complexo que outro, podendo levar a um falso resultado.

A pesquisa qualitativa apresentou indícios de que os alunos ficaram motivados durante as sessões com os jogos. Além disso, os alunos também tiveram interesse em jogar novamente, uma vez que o jogo possui um ranking dos melhores jogadores. Este tipo de motivação poderá auxiliar na fixação do conteúdo da Engenharia de Requisitos. Ainda, a avaliação qualitativa também indicou características do jogo que podem ser melhoradas em versões futuras, tais como o nível de dificuldade, apontada por alguns alunos como elevada e também fornecer mais informações sobre o conteúdo da Engenharia de Requisitos.

\section{Referências}

Alexander, M. and Beatty, J. "Effective Design and Use of Requirements Engineering Training Games". REET, 2008.

Anderson, L. W. "Rethinking Bloom's Taxonomy: implication for testing and assessment". Columbia: University of South Carolina, 1999. (Report n. MF01/PC01).

El-shamy, S. "Training Games: Everything You need to Know About Using Games to Reinforce Learning”. Stylus Publishing, Sterling, VA, 2001.

Ferguson, R. and Lami, G. "An Empirical Study on The Relationship Between Defective Requirements and Test Failures". 30th IEEE, 2006.

Fernandes, M., Machado, R. and Seidman, S. "A Requirements Enginerring and Management Training Course for Software Development Professionals". 22th Conference on Software Engineering Education and Training, 2009.

Goncalves, R. Q. and Thiry, M. "Development of a game to support the teaching of requirements engineering: The Requirements Island". In: IX SBGames, 2010, 
Florianópolis, SC. SBC - Proceedings of SBGames 2010. Porto Alegre, RS : SBC, 2010. p. 358-361.

Greeno, J., Collins, A. and Resnick, L. "Cognition and learning”. Macmillan, New York , 1996.

Knauss, E., Schneider, K. and Stapel, K. "A Game for taking requirements engineering more seriously". Third International Workshop on Multimedia and Enjoyable Requirements Engineering (MERE 08), Spain, Barcelona, 2008.

Kochanski, D., Wangenheim, C. and Thiry, M. "Empirical evaluation of an educational game on software measurement". Empirical Software Engineering. 2009.

Levin, J. and Fox, J. "Elementary Statistics in Social Research". Allyn \& Bacon, 2006

Romero, M., Vizcaíno, A. and Piattini, M. "A Simulator for Education and Training in Global Requirements Engineering: a Work in Progress". Eighth IEEE International Conference on Volume, 2008.

Smith, R. and Gotel O. "RE-O-Poly: A Customizable Game to Introduce and Reinforce Requirements Engineering Good Practices". Departament of Computer Science, Pace University, New York, 2008.

Soares, A. "Introdução, Identificação e Análise em Engenharia de Requisitos". 2005.

Sommerville, I. “Engenharia de Software ”. PEARSON Addison Wesley, vol. 8, 2007.

Sommerville, I. and Kotonya G. "Requirements Engineering: Processes and Techniques". 1998.

Thiry, M., Zoucas, A. and Goncalves, R. Q. "Promovendo a Aprendizagem de Engenharia de Requisitos de Software Através de um Jogo Educativo" In: SBIE'10 21o SIMPÓSIO BRASILEIRO DE INFORMÁTICA NA EDUCAÇÃO, ISSN: 21764301, João Pessoa - PB, 23 a 26 de Novembro de 2010

Thiry, M., Zoucas, A., Goncalves, R. Q. and Salviano C. "Aplicação de jogos educativos para aprendizagem em melhoria de processo e Engenharia de Software". In: Workshop Anual do MPS, 2010, Campinas, SP. Anais do VI WAMPS 2010. Campinas, SP : Softex, 2010. v. 1. p. 118-127. 